\title{
INTEGRATED SYSTEM FOR MONITORING AND RECOGNIZING STUDENTS DURING CLASS SESSION
}

\author{
Mohammad A. Alia, Abdelfatah Aref Tamimi and Omaima N. A. AL-Allaf \\ Faculty of Science and Information Technology - Al Zaytoonah University of Jordan, \\ P.O.Box: 130 Amman (11733) Jordan
}

\begin{abstract}
In this paper we propose a new student attendance system based on biometric authentication protocol. This system is basically using the face detection and the recognition protocols to facilitate checking students' attendance in the classroom. In the proposed system, the classroom's camera is capturing the students' photo, directly the face detection and recognition processes will be implemented to produce the instructor attendance report. Actually, this system is more efficient than others student attendance methods since the detection and the recognition are considered to be the best and fastest method for biometric attendance system. Regarding to the students and instructor sides, the system is working without any preparation and with no more effort.
\end{abstract}

\section{KEYWORDS}

Face Recognition, Face Detection, Monitoring, Attendance System, and System Access Control.

\section{INTRODUCTION}

Today, Face detection and recognition are very advanced in terms of computer authentication technology. The technology of student attendance system is used to support the teacher for checking his/her students' attendance in modern way. Face detection and recognition are considered to be the best and fastest method for biometric attendance system. They are secure to use and working without any preparation since the camera can direct capture the student faces, and then the report of attendant students will be produced immediately with no more effort.

Designing a trustworthy student attendance system based on face detection and recognition is considered the faster and optimal way to manage the records for students' attendance in institutes. Furthermore, any business organization or educational institution has to maintain the attendance of students or employees for effective functioning of business records. The important key of this paper is to design a better student attendance system with ease interface and accurate results.

In the proposed attendance system, two individual authentications protocols were analysed in terms of security properties. These protocols are the face detection protocol and face recognition protocol. However, several potential weaknesses were discovered in these authentication

DOI : 10.5121/ijma.2013.5604 
The International Journal of Multimedia \& Its Applications (IJMA) Vol.5, No.6, December 2013

protocols. The weaknesses are defined according to the problem of the face detection or recognition algorithms. These weaknesses include: problems resulting from human unreliability in computer systems, algorithm accuracy, algorithm process time, and denial of service. Therefore, the expectation of a well designed implementation and deployment may be able to mitigate or even eliminate the impact of these weaknesses.

\section{FACE DETECTION AND RECOGNITION SCHEMES}

Face detection and recognition schemes are a complementary parties scheme, each party is a complement to the other. Meanwhile, each party can work individually depending on regular system. However, Face detection technology is a computer technology that is based on learning algorithms to allocate human faces in digital images. As shown by Figure 1, face detection algorithm determines the facial features and ignores anything else in the digital images[1]. Furthermore, many face detection techniques are proposed such as; Viola and Jones face detection [2], face detection based Adaboost [3], semi-supervised learning for facial expression recognition [4], and etc.

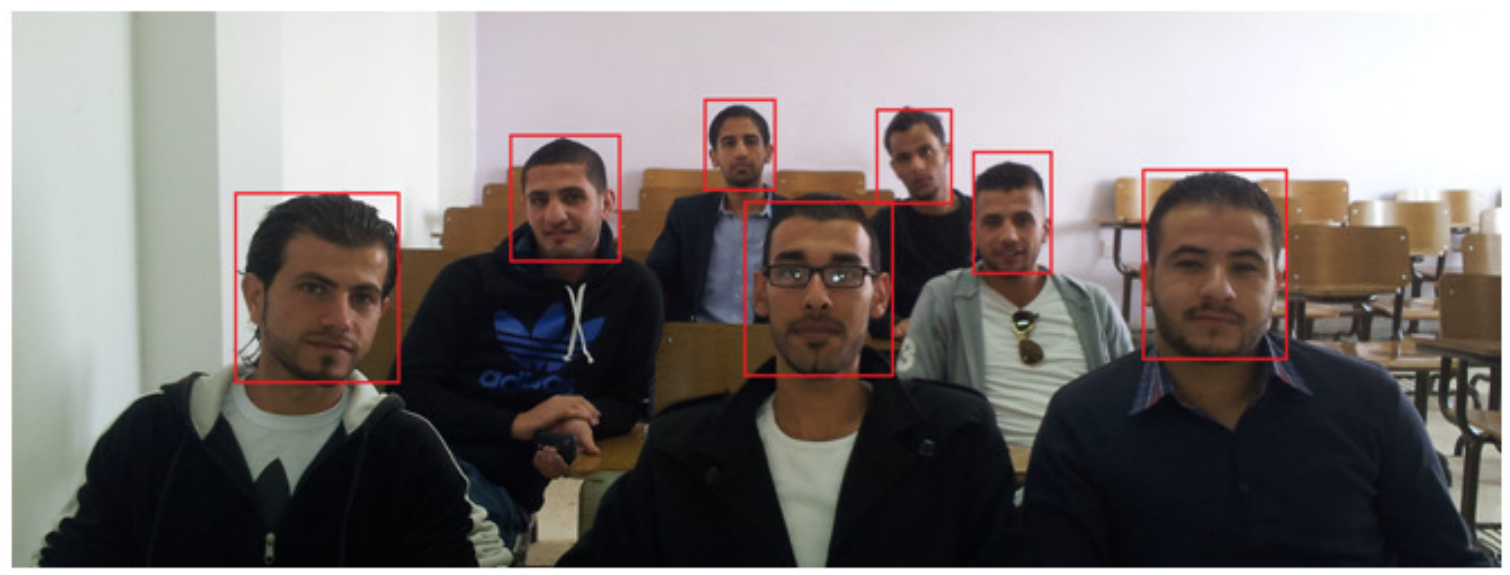

Figure 1: Face Detection

In the other hand, face recognition (refer to Figure 2) is a natural biological authentication process technology according to the cognitive rule of human beings. It is used to identify any given face image using the main features of this face [5]. Normally, face recognition process works after face detection process to identify the detected face by comparing the detected faces with the stored faces images. In authentication process, face recognition algorisms are more complex and secure than other authentication algorithms such as fingerprint authentication algorithm. Comparing face recognition to other techniques, face recognition is featured as: accurate and fast Identification, and high usability and security however. Whereby, many face recognition algorithms have been presented and based on different artificial neural network technologies such as: feed forward back propagation neural network (FFBPNN), cascade forward back propagation neural network (CFBPNN), function fitting neural network (FitNet) and pattern recognition neural network (PatternNet) algorithms [5]. Among these are: Soon and Seiichi [6], While Volkan [7], Weihua and WeiFu [8], and etc. 


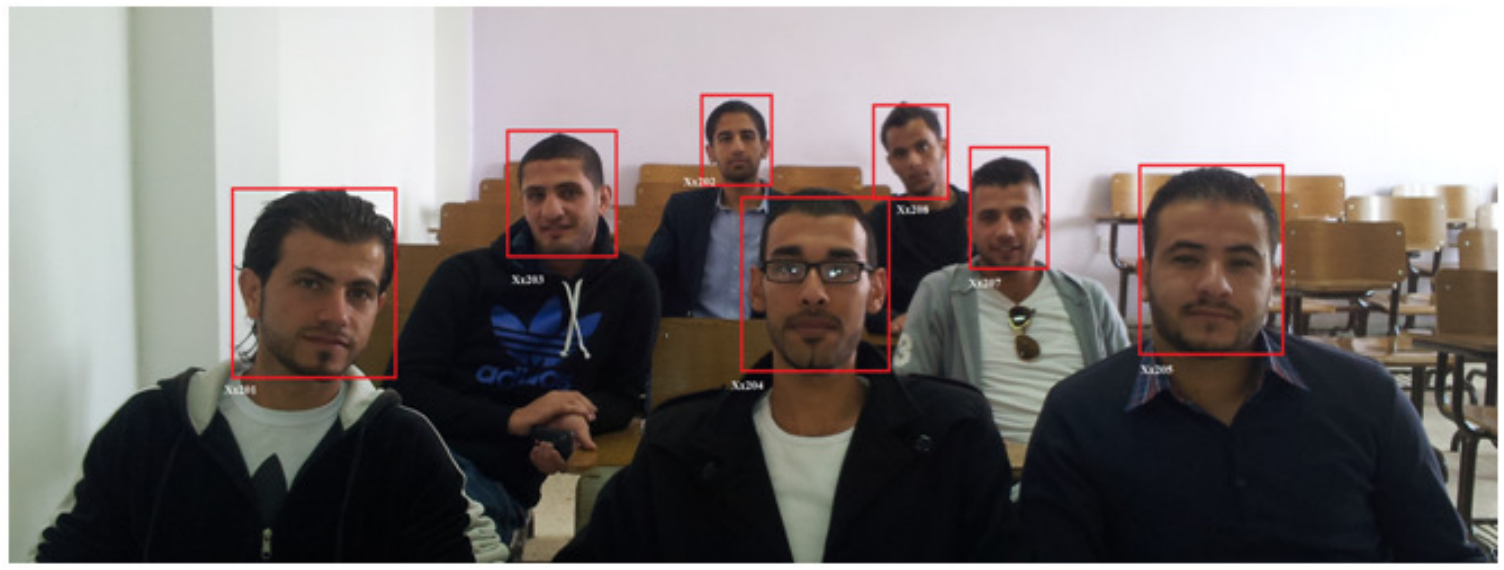

Figure 2: Face Recognition

\section{IDENTIFICATION AND AUTHENTICATION}

Identification and authentication [9] (I\&A) is an important field in system access control process. Identification and authentication is the process that can be used to identify and verify the entity on the system. In multi-user system, the users must identify themselves firstly, and then the system will authenticate the identity before using the system. Therefore, the identification and authentication processes can be implemented by the following three classical techniques:

1. Something you know: password.

2. Something you have: smart card.

3. Something you are: face recognition, fingerprint, voice, retina, or iris characteristics.

\section{ATTENDANCE SYSTEMS}

In general manual attendance record is very limited. It is well enough for small number of students. As well as, manual system causes some disadvantages such as time waste over students' responses, waste of paper, and etc.

Student attendance system is a management system used to manage and report student attendance in the classroom. It can monitor student attendance, generate reports with school, district, and state attendance information. From the other hand, attendance system is not only dedicated for educational institutions, since it can be applied in different organizations (refer to Figure 3, 4, and 5). In fact, organizations use time based attendance systems to manage their employees' records when employees start and stop work. However, the student attendance system provides many benefits to the educational organizations by controlling all students attendance hours in the classroom. 
The International Journal of Multimedia \& Its Applications (IJMA) Vol.5, No.6, December 2013

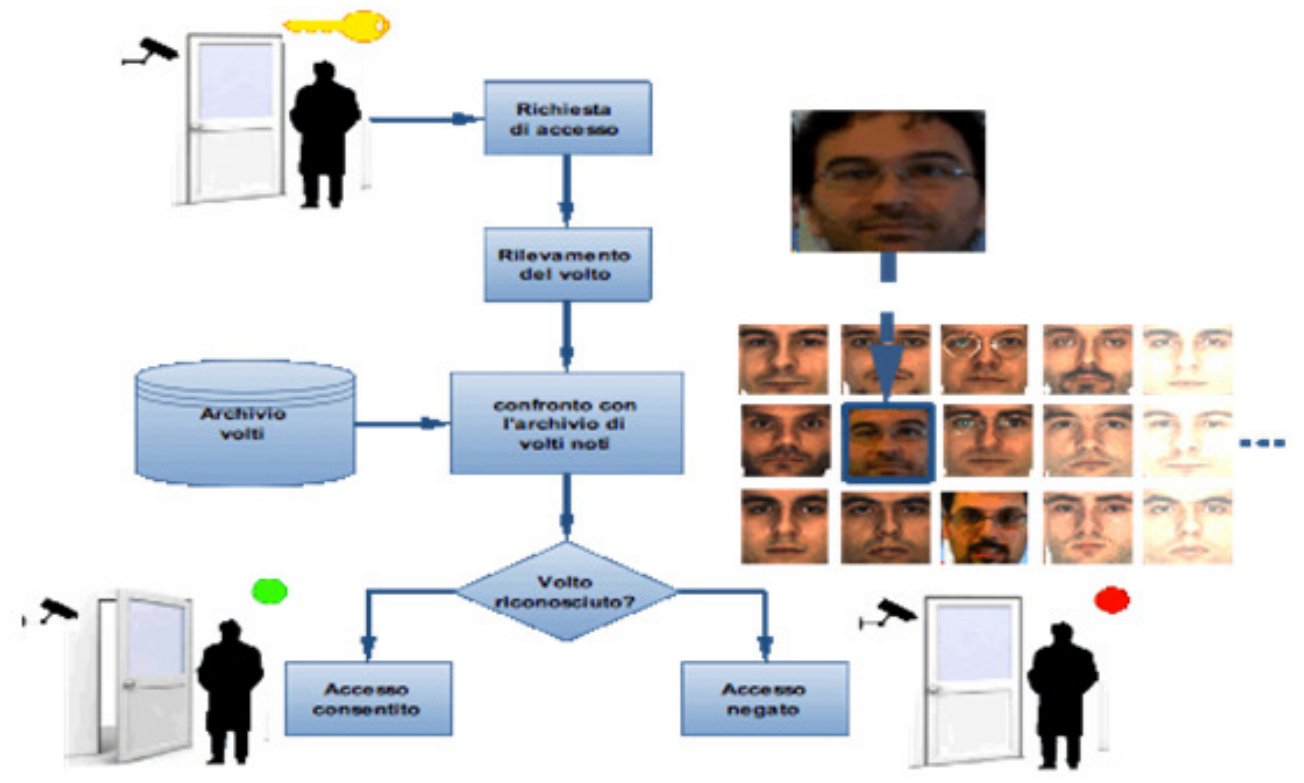

Figure 3: attendance system based single face detection[10]

Many automatic attendance systems have been proposed by using fingerprint verification technique. Among these are the Efficient Automatic Attendance System using Fingerprint Verification Technique [11], and the Attendance Monitoring System Using Biometrics [12], and the Student Attendance System Based On Fingerprint Recognition and One-to-Many Matching [13].

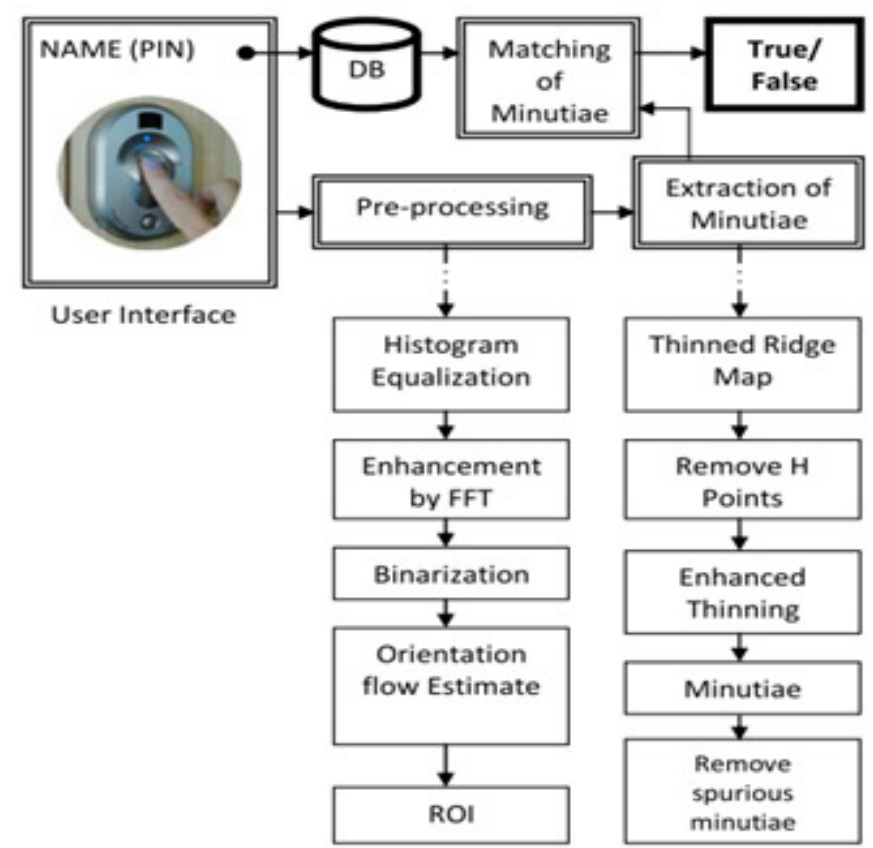

Figure 4: An Attendance Monitoring System Using Biometrics [12] 
The International Journal of Multimedia \& Its Applications (IJMA) Vol.5, No.6, December 2013

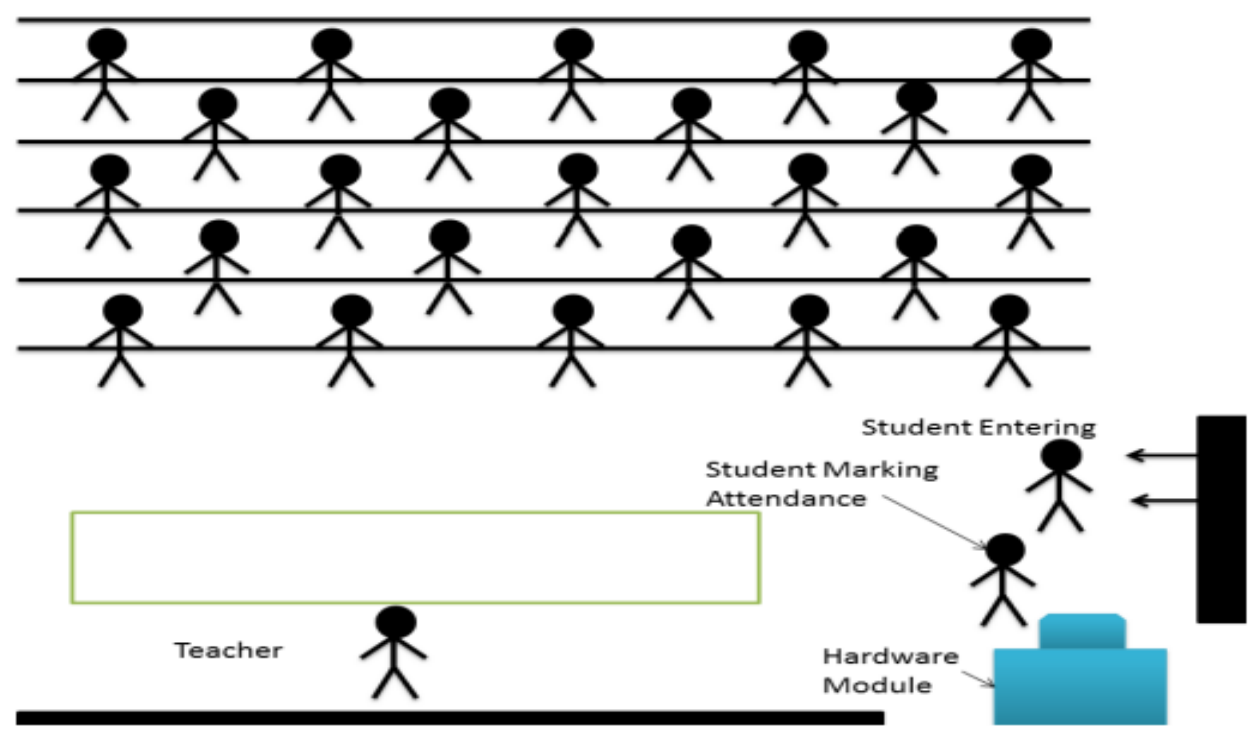

Figure 5: student attendance system [13]

\section{The Proposed Integrated System For Monitoring AND RECOGNIZING STUDENTS DURING ClASS SESSION}

As mentioned earlier, many previous studies on student attendance system have been done and focused on facilitating the attendance method. These methods are basically depend on the standard variant authentication schemes such as finger/ hand print, cards, or etc.

In this paper, the proposed attendance system is based on faces detection and recognition processes. Therefore the proposed system is minimizing the instructor' exhaustion since the instructors can check their student's attendance by using their own PCs and the required time to collect and analyse the final results. However, the proposed system is based on face detection and recognition authentication methods. Whereby, the face recognition method is the most natural biological features recognition technology according to the cognitive rule of human beings.

Generally, the proposed system describes three steps for students attendance system (refer to Figures 6). These steps are: the system access control step, the face detection and recognition step, and collecting data step.

\section{System Access Control Step}

As mentioned earlier, system access control process is one of the cryptographic services, which is used to authenticate the instructor in the student database server. As well the instructor can access his/her students list. This process is the first step in the proposed students' attendance system that prepares the instructor to be authorized in the advanced students' attendance process in the classroom. Therefore, the system access control includes Identification phase which identify the instructor on the system; and Authentication phase which authenticate the instructor on his classroom's students. 
The International Journal of Multimedia \& Its Applications (IJMA) Vol.5, No.6, December 2013

Moreover, the whole student attendance system is clarified in Figure 6 from the beginning to the end. The instructor login to the attendance system by using his/her ID and password. Once the students enter their classroom, the classroom camera will record and capture the students' photos as shown by Figure 6, Step 1. In Figure 6 Step 2 and 3, the instructor should request the faces detection and recognition phase to check the attendant and absent students in his/her classroom immediately.

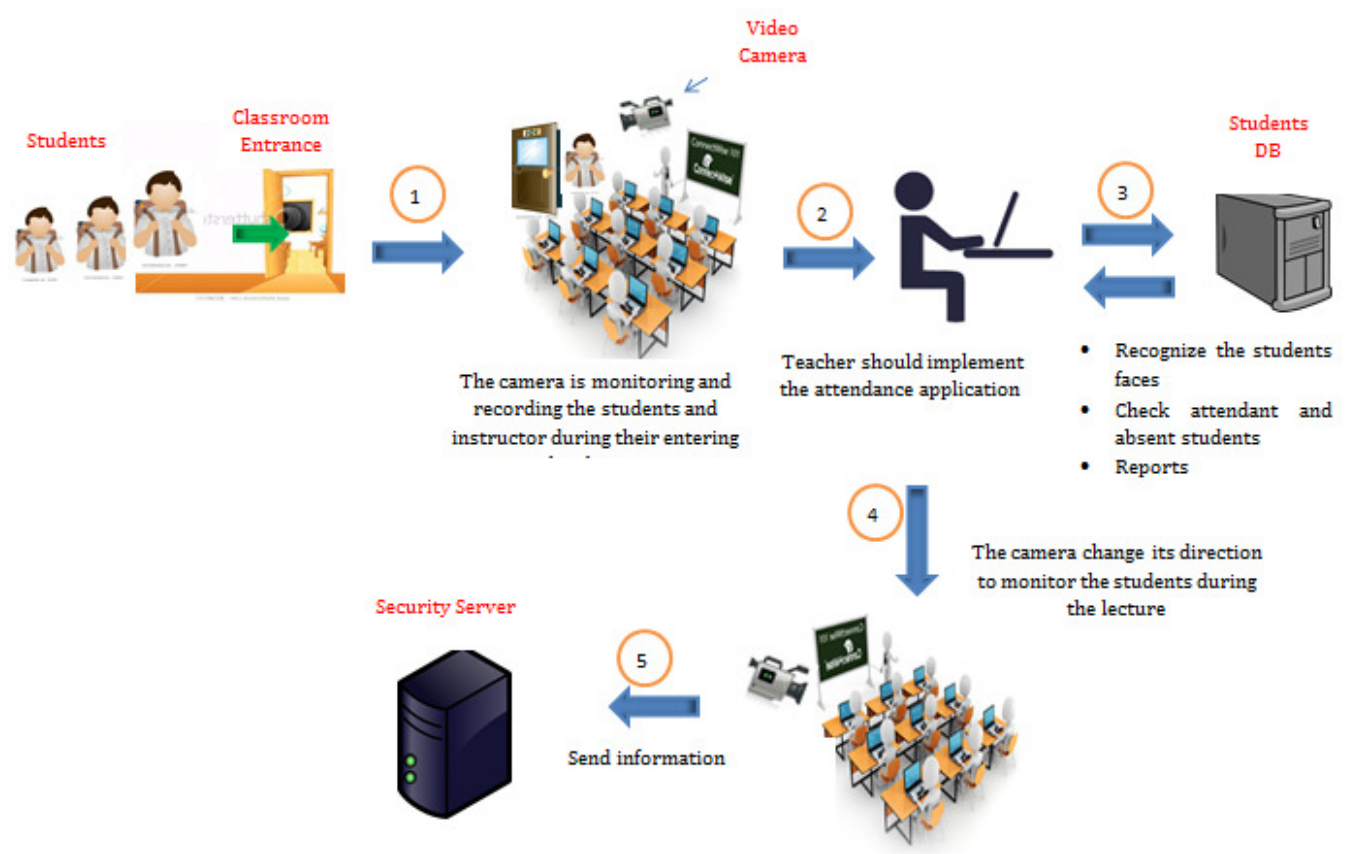

Figure 6: the proposed system

\section{Face Detection and Recognition Step}

Typically, the system process is very critical since the face detection process is starting after capturing the students' photos' by classroom's camera, as shown by Figure 7. However, the classroom's instructor should upload the student photo, as well the system will filter the student photo to detect all students' faces (refer to Figure 7). Figure 7 Step 3 shows that the students' faeces are already detected since the attendance list is open and recognize all the attendees. However, the system enables the instructor to drag and drop the attendant student photo in case his/her photo is not detected or recognized by the system as shown by Figure 7. 
The International Journal of Multimedia \& Its Applications (IJMA) Vol.5, No.6, December 2013
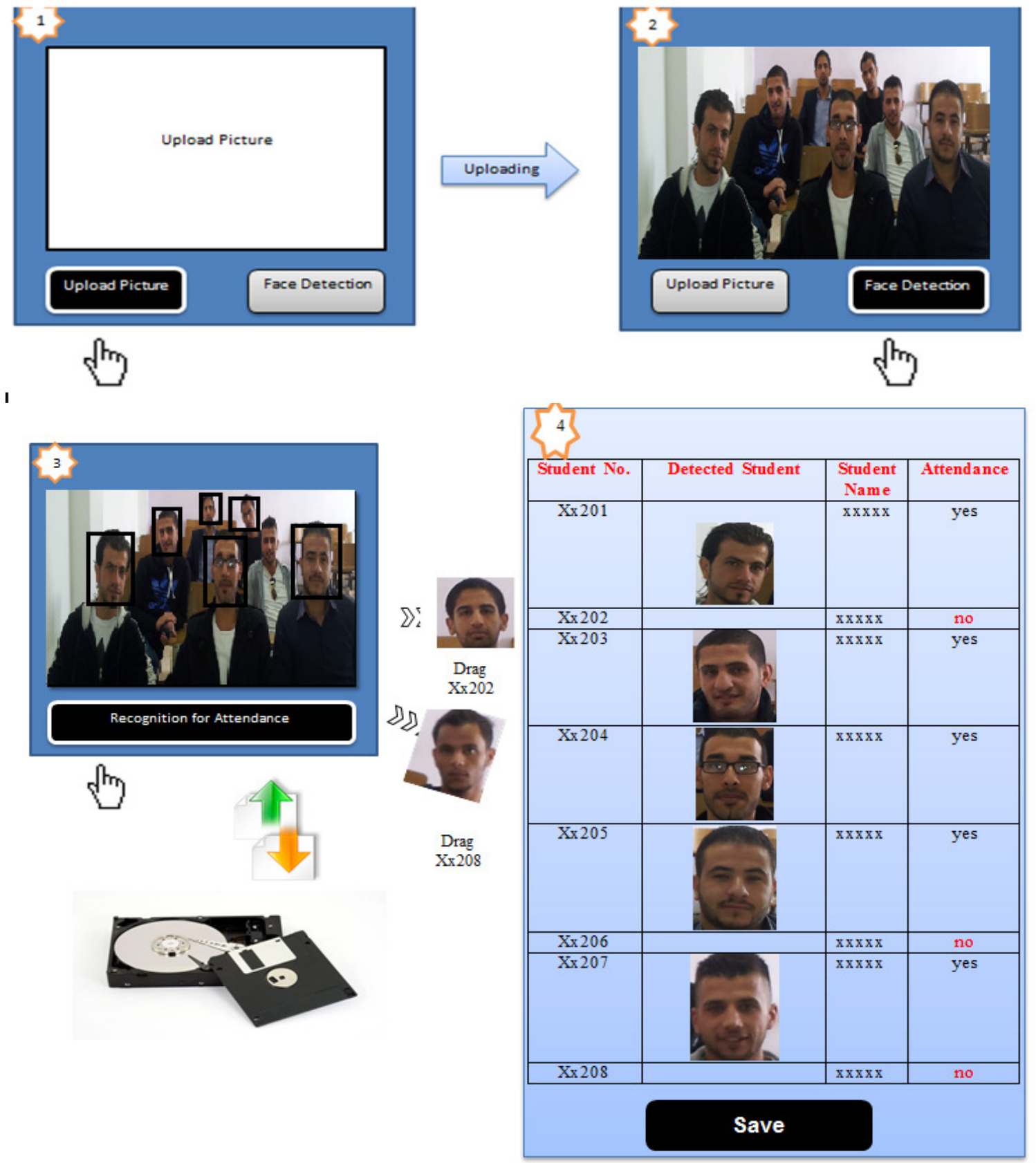

Figure 7: the proposed attendance system

\section{Collecting Data Process}

The third step in the proposed method is to collect data and produce the attendance report. After the detection and recognition process, the data will be transmitted to the attendance system database, whereby the instructor can immediately produce his/her students' attendance report (refer to Figure 7). 
The International Journal of Multimedia \& Its Applications (IJMA) Vol.5, No.6, December 2013

\section{CONCLUSION}

This paper has shown the possibility of establishing student attendance system based on face detection and recognition authentication scheme. The system allows the instructor to check his/her student attendance automatically by using personal computer (PC) without any extra cost and effort. As new technology for student attendance system, this system is proposed to replace the previous biometric attendance system, since attendance report can be created by the classroom camera. As well as, the proposed system needs only the basic requirements such as; camera, PC, and local network.

\section{ACKNOWLEDGMENT}

The authors would like to thank Al Zaytoonah University of Jordan for supporting this study.

\section{REFERENCES}

[1] I. Marqu'es, and M. Gra na. Face Recognition Algorithms. Proyectos Fin de Carrera, Universidad Carlos III de Madrid.2010.

[2] P. Viola , and M. Jones.Rapid object detection using a boosted cascade of simple features. Accepted Conference On Computer Vision And Pattern Recognition, 2001.

[3] Yan-Wen Wu , and Xue-Yi Ai. Face Detection in Color Images Using AdaBoost Algorithm Based on Skin Color Information. First International Workshop on Knowledge Discovery and Data Mining, 2008.

[4] I. Cohen , N. Sebe , F. G. Cozman ,Thomas S. Huang . Semi-Supervised Learning for Facial Expression Recognition. Proceedings of the 5th ACM SIGMM international workshop on Multimedia information retrieval. ACM New York, NY, USA Pages 17 - 22.

[5] O. N. A. AL-Allaf, A. Aref Tamimi , and M. A. Alia. Face Recognition System Based on Different Artificial Neural Networks Models and Training Algorithms. International Journal of Advanced Computer Science and Applications, Vol. 4, No. 6, 2013.

[6] S. L.Toh and O. Seiichi. A Face Recognition System Using Neural Networks with Incremental Learning Ability. Proceeding of the 8th Australian and New Zealand Conf. on Intelligent Information Systems. pp.389-394. 2003.

[7] A. Volkan . Face Recognition Using Eigenfaces and Neural Networks. Master of Science Thesis, The Graduate School of Natural And Applied Sciences. The Middle East Technical University. 2003.

[8] W. Weihua and W. WeiFu . A Gray-Scale Face Recognition Approach. Second International Symposium on Intelligent Information Technology Application, 978-0-7695-3497-8/08, IEEE computer society. 2008. DOI=10.1109/IITA.2008.101.

[9] R. Lehtinen,. Computer Security Basics, 2nd Edition, O'Reilly. 2006. ISBN-10: 0-596-00669-1.

[10] Eye-tech. http://www.eye-tech.it/index.php?lang=en

[11] C. Saraswat and A. Kumar. An Efficient Automatic Attendance System using Fingerprint Verification Technique. Chitresh Saraswat et al. / (IJCSE) International Journal on Computer Science and Engineering, Vol. 02, No. 02, Pp.264-269. 2010.

[12] S. Rao, and K. J. Satoa. An Attendance Monitoring System Using Biometrics Authentication. International Journal of Advanced Research in Computer Science and Software Engineering. Volume 3, Issue 4, pp. 379-383. 2013

[13] R. Mishra and P. Trivedi. Student Attendance System Based On Fingerprint Recognition and One-toMany Matching. Department of Computer Science and Engineering National Institute of Technology Rourkela Rourkela-769 008, Orissa, India. 2011. 